\title{
THE RING UNCEMENTED POLYETHYLENE CUP IN THE ABNORMAL ACETABULUM
}

\author{
D. NUNN
}

From Redhill Hospital

\begin{abstract}
The results of using the Ring uncemented polyethylene-to-metal (UPM) prosthesis in patients with protrusio acetabuli or dysplasia are reported. Fifty-four cases of protrusio were analysed and a modified method of measuring the deformity is described; after operation, remodelling of the medial wall had improved, on average, by $29.6 \%$.

For the dysplastic acetabulum the main difficulty is the lack of lateral support for the cup. In order to test the hypothesis that this support improves with an uncemented prosthesis, 38 hips with an average follow-up of 31 months were studied. Lateral support, measured radiographically, showed an average increase of $21 \%$. The remodelling effect may be attributed to the biomechanical design of the prosthesis.
\end{abstract}

Acetabular protrusion makes it difficult to insert an uncemented prosthesis. With the Ring prosthesis, however, this difficulty can be overcome since the design of the cup allows load to be transmitted to the weightbearing line of the pelvis. This, and the absence of cement, may then lead to resolution and remodelling of the pelvic defect, according to Wolff's law.

The dysplastic acetabulum presents a different problem, namely the lack of lateral support for the prosthesis. With a cemented hip, the cement may have insufficient support, while with an uncemented hip the prosthesis itself may be left unsupported.

It has already been shown by Bertin et al. (1985) that with an uncemented cup a zone of sclerosis develops adjacent to the prosthesis and that this process reaches a plateau at about one year. It has also been demonstrated that protrusio can resolve with the metal Ring prosthesis (Sharp, Porter and Duke 1984).

\section{PATIENTS AND METHOD}

Between July 1980 and December 1983 total hip replacements were performed on 61 patients (63 hips) with protrusio acetabuli, and on 42 patients (48 hips) with dysplasia of the acetabulum. All the operations were carried out by one surgeon.

D. Nunn, FRCS Ed, Orthopaedic Registrar Orthopaedic Research Unit, Redhill General Hospital, Earlswood Common, Redhill, Surrey RHI 6LA, England.

Requests for reprints should be sent to Mr D. Nunn, 15 Thurlow Road, Hanwell, London W7 2JG, England.

(C) 1987 British Editorial Society of Bone and Joint Surgery $0301-620 \mathrm{X} / 87 / 5160 \$ 2.00$
The Ring UPM cup is a polyethylene offset cone with an internal diameter of $32 \mathrm{~mm}$. It is available in 45 , 50,55 and $60 \mathrm{~mm}$ external diameters. Initial fixation is obtained via a Freeman finned osseous peg, but stability ultimately depends on the wedge compression effect of the cone-shaped cup inserted into the reciprocally prepared acetabulum.

The patients were reviewed clinically and radiographically every year, and the records of 54 protrusio hips (52 patients) and of 38 dysplastic hips (32 patients) were available for review.

Technique of operation. The details of the operation have been reported elsewhere (Ring 1983), but essentially the insertion depends on locating the iliopubic bar of bone; a guide-wire is introduced via a jig and this aligns the reamers (and therefore the cup) in the weight-bearing line of the pelvis.

With protrusio, the guide-wire is directed more to the lateral side of the acetabulum than to the medial. The acetabulum is then reamed to produce a concentric cone. After reaming, it may be found that the cone, and therefore the support of the cup, is deficient medially, and a bone graft may be required. The femoral component is inserted in standard fashion. Postoperatively the patient is mobilised as usual, and no special precautions are necessary.

With a dysplastic acetabulum, the guide-wire is inserted as far medially as possible. The acetabulum is then reamed and the cup is punched home. It may be found that the lateral support for the cup is deficient, and the defect can be grafted with the bone paste produced by reaming, but this was done in only one patient in the present series. (More recently a block of bone has been used in younger patients.) Postoperatively, the patient is 


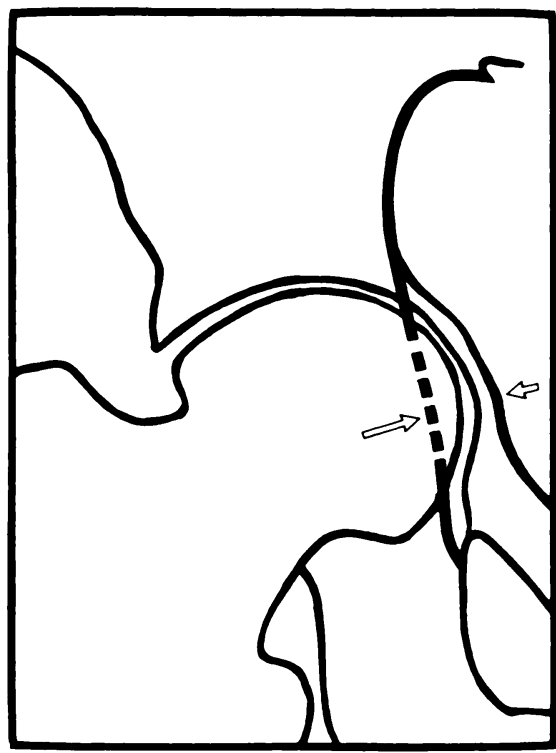

Fig. 1

Protrusion is measured in millimetres from the dotted line to the most medial portion of the protrusion.

mobilised in the usual manner unless a bone graft has been performed, in which case he or she spends a few days longer in traction and rather longer on crutches.

Radiographic measurements. Protrusio. Measurements were carried out on standard anteroposterior views of the pelvis. Sotelo-Garza and Charnley (1978) described a method of measurement from a projected "true" pelvic line to the most medial point of the protrusion. In our unit, however, we found it difficult to draw this line in a reproducible way, and therefore used a line of the medial wall of the pelvis, shown dotted in Figure 1. From this line, which is always visible in the absence of radiopaque cement, the distance to the most medial point of the protrusion is measured. All measurements were standardised for magnification by multiplying the measurements by the actual diameter of the femoral head $(32 \mathrm{~mm})$, and then dividing this figure by the measured diameter.

The results were then treated in two ways. Firstly, the measurements were graded in order to allow comparison with the Wrightington figures (Sotelo-Garza and Charnley 1978). Secondly, the difference between the initial figure and that from the last review was expressed as a percentage of the original in order to indicate the degree of pelvic remodelling. An example of the kind of resolution which may occur is shown in Figures 2 to 4 .

Dysplasia. Before reviewing the radiographs it was necessary to devise a reliable method of measurement which allowed for rotation and magnification. Films were taken of a dry hip in varying degrees of rotation of the pelvis. The centre-edge (CE) angle and the width of the ilium at its narrowest point above the acetabulum were determined (Fig. 5), and the $\mathrm{CE}$ angle was then divided by the width of the ilium measured in millimetres; this is called the index of dysplasia. It was found that this index was constant if the pelvis was inclined $15^{\circ}$ either side of the horizontal plane, and it was thought that this range was unlikely to be exceeded in standard anteroposterior radiographs of the pelvis. Measurements were then made on the radiographs of the patients. The effects of magnification were compensated for in the same way as for the protrusio figures.

The difference between the first postoperative index

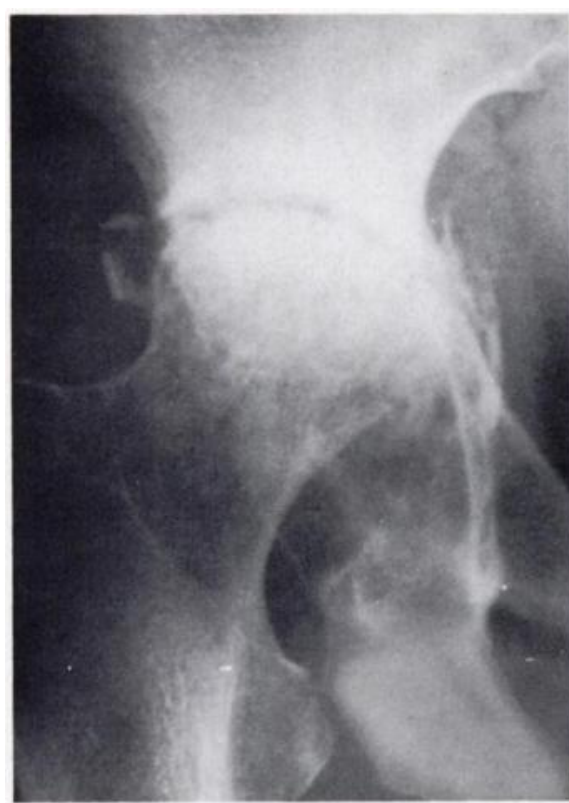

Fig. 2

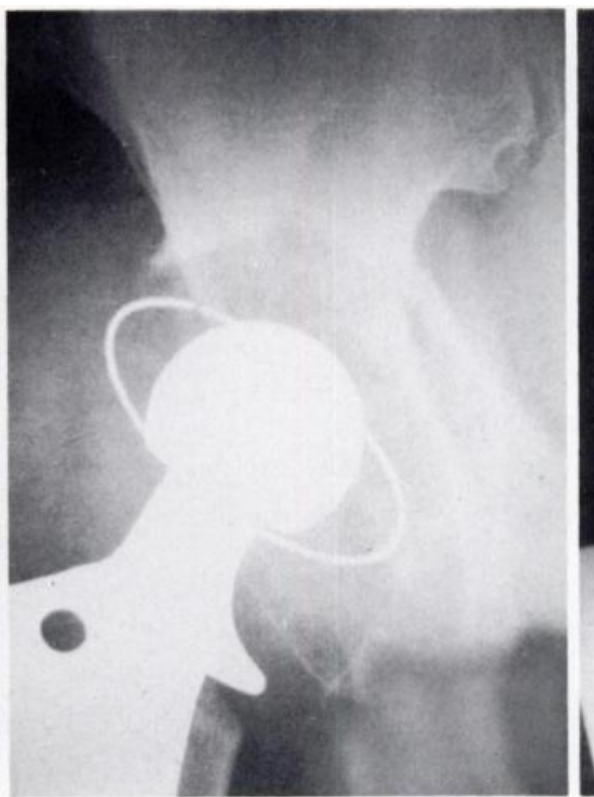

Fig. 3

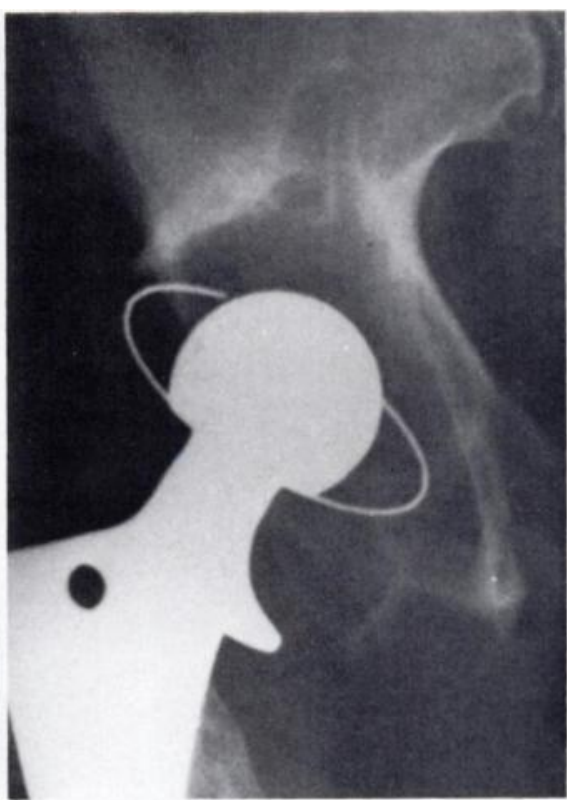

Fig. 4

Serial radiographs showing resolution of acetabular protrusion after a total hip replacement using a Ring UPM prosthesis. 


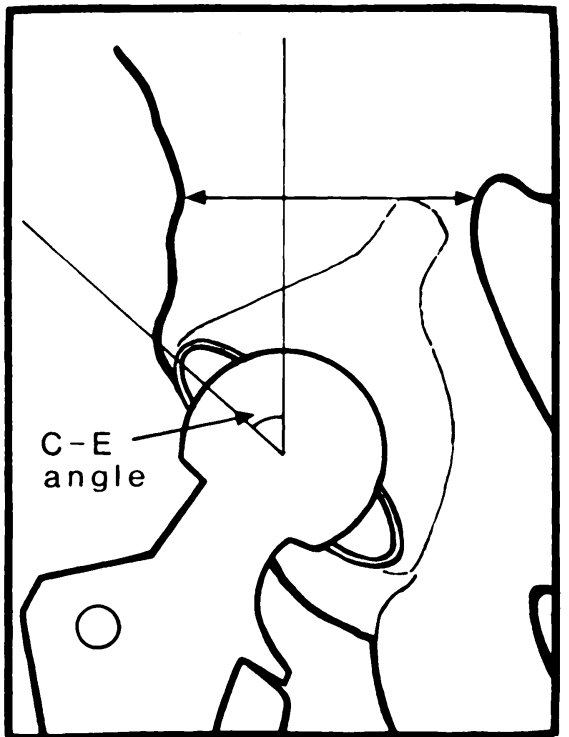

Fig. 5

Diagram showing the method of measuring the index of dysplasia. The formula for this calculation is : index $=\mathrm{CE}$ angle width of ilium $(\mathrm{mm})$

and the one at the latest review was expressed as a percentage of the original index, in order to indicate improvement in the lateral cover of the cup. The results were also graded (Table I). Improvement in lateral support for the acetabular cup is shown in Figures 6 to 8.

\section{RESULTS}

Protrusio. There were 54 hip replacements in 52 patients, and follow-up ranged from 12 to 60 months (mean 30 months). The results of this series were graded in the
Table I. Grading of acetabular dysplasia

\begin{tabular}{ll}
\hline Grade & CE angle \\
\hline Severe & $15^{\circ}-25^{\circ}$ \\
Moderate & $26^{\circ}-35^{\circ}$ \\
Mild & $>35^{\circ}$ \\
\hline
\end{tabular}

Table II. Improvement in the grade of acetabular protrusion in 54 hips*

\begin{tabular}{llll}
\hline & & \multicolumn{2}{l}{ Number of hips } \\
\cline { 3 - 4 } Grade & Protrusion (mm) & $\begin{array}{l}\text { Initial postoperative } \\
\text { radiograph }\end{array}$ & At latest review \\
\hline 0 & None (0) & 0 & 1 \\
I & Mild (1-5) & 8 & 22 \\
II & Moderate (6-15) & 42 & 30 \\
III & Severe (>15) & 4 & 1 \\
\hline
\end{tabular}

- Sotelo-Garza and Charnley 1978

same way as Sotelo-Garza and Charnley's 1978 series (Table II), and it was found that 21 hips improved by at least one grade.

The percentage of improvement of the degree of protrusion is shown in Table III. The overall average improvement was $29.6 \%$; in other words, there was an average decrease in the degree of protrusion of about one-third. One patient showed complete resolution. Six patients showed deterioration from $1.7 \%$ to $25.5 \%$, that is, the acetabular defect worsened; of these six, two had rheumatoid arthritis, and in one of these there was definite evidence of cup loosening.

Dysplasia. There were 38 hip replacements (32 patients)

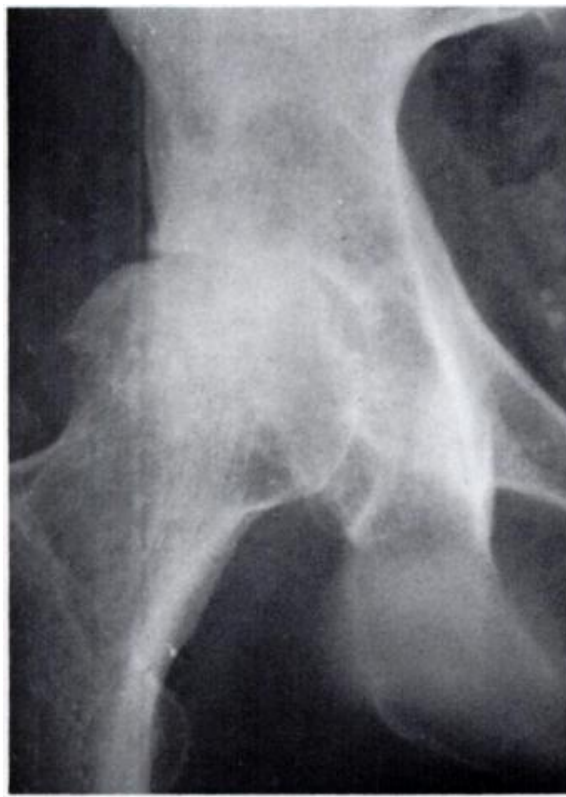

Fig. 6

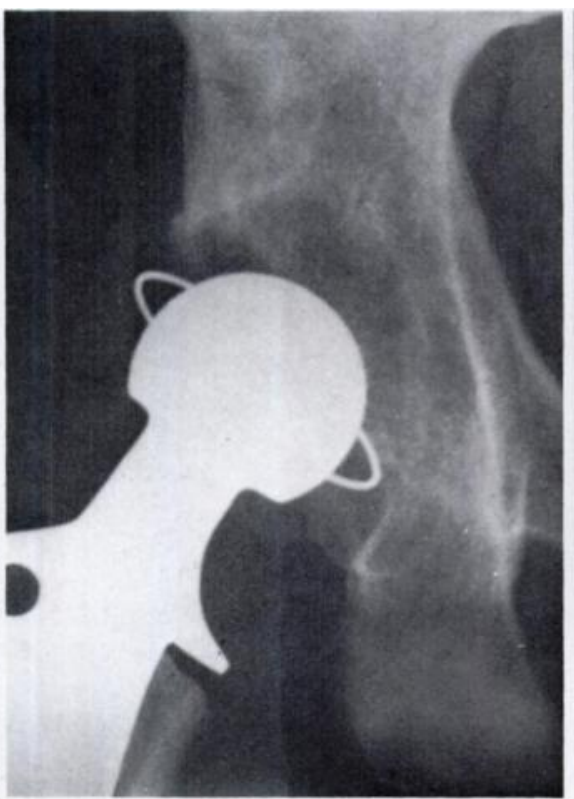

Fig. 7

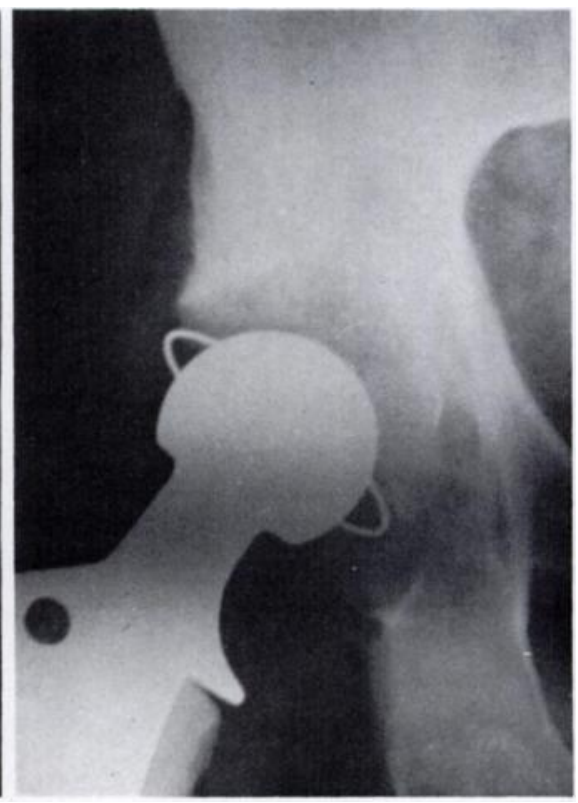

Fig. 8

Serial radiographs showing improvement in lateral support of the acetabular cup. 
Table III. Percentage improvement of protrusion in 48 of 54 hips* $^{*}$

\begin{tabular}{cc}
\hline $\begin{array}{l}\text { Percentage } \\
\text { improvement }\end{array}$ & $\begin{array}{c}\text { Number } \\
\text { of hips }\end{array}$ \\
\hline $0-10$ & 7 \\
$11-20$ & 6 \\
$21-30$ & 6 \\
$31-40$ & 10 \\
$41-50$ & 5 \\
$51-60$ & 7 \\
$61-70$ & 5 \\
$71-80$ & 1 \\
$81-90$ & 0 \\
$91-100$ & 1 \\
Total & 48 \\
\hline
\end{tabular}

- In six patients the acetabular defect worsened
Table IV. Percentage improvement of the index of dysplasia in 29 hips* $^{*}$

\begin{tabular}{ll}
\hline $\begin{array}{l}\text { Percentage } \\
\text { improvement }\end{array}$ & $\begin{array}{l}\text { Number } \\
\text { of hips }\end{array}$ \\
\hline $0-10$ & 9 \\
$11-20$ & 6 \\
$21-30$ & 1 \\
$31-40$ & 4 \\
$41-50$ & 2 \\
$51-60$ & 2 \\
$61-70$ & 2 \\
$71-80$ & 2 \\
$81-90$ & 0 \\
$91-100$ & 0 \\
$>100$ & 1 \\
\hline
\end{tabular}

* In nine hips the dysplasia worsened

and the mean follow-up was 31 months (range 12 to 60 months). The average age was 66 years (range 36 to 84 years). One of the patients had had the lateral edge of the acetabulum augmented with a bone-paste graft. The percentage improvement of the dysplastic index is shown in Table IV.

In nine patients there was deterioration of the lateral support, indicated by a negative value (range -1.2 to -22.1 ). One patient showed an improvement of $135 \%$. The average overall improvement was $21.5 \%$. There was a strong negative correlation $(-0.64)$ between the age of the patient and the percentage improvement, that is, the younger patients showed greater improvement; this accounts for the scatter of the results. The results of grading according to an improvement in the $\mathrm{CE}$ angle is shown in Table $\mathrm{V}$.

Table V. Improvement in the grade of the CE angle

\begin{tabular}{|c|c|c|}
\hline \multirow[b]{2}{*}{ Grade } & \multicolumn{2}{|c|}{ Number of hips } \\
\hline & $\begin{array}{l}\text { Initial } \\
\text { postoperative } \\
\text { radiograph }\end{array}$ & At latest review \\
\hline Severe & 9 & 5 \\
\hline Moderate & 15 & 9 \\
\hline Mild & 14 & 24 \\
\hline
\end{tabular}

\section{DISCUSSION}

Protrusio. Twenty-one hips in the group with acetabular protrusion improved by one grade after a Ring UPM prosthesis was inserted. This shows that the load characteristics of this prosthesis are such that remodell-

ing of the bone stock does occur: the force of weightbearing is transmitted concentrically from the offset cone of the cup to the reciprocally shaped acetabulum along the correct anatomical line. The response of the loaded bone is seen as an area of sclerosis at the cup bone interface. The medial wall of the acetabulum, which was weight-bearing before operation, is unloaded by this tvpe of replacement and is, in consequence, resorbed. This process continues until the remodelled bone abuts on to the medial side of the prosthesis, comes under load. and consequently scleroses and, presumably, strengthens. It may even be that, should revision be necessary for femoral loosening, the pelvic bone stock will be better than at the primary operation.

Bertin et al. (1985) also found that a zone of sclerosis develops adjacent to uncemented acetabular prostheses, and that this process reached a plateau at about one year. In our study, those patients with the longest follow-up did not seem to improve further after about 30 months. The same authors also reported histological studies which showed that the cup-bone interface under load consists of a thin layer of fibrous tissue with collagen fibres running parallel to the surface, as well as metaplastic cartilage and new bone, indicating the bone-forming potential of this layer.

Ranawat and Zahn (1986), in describing their series of 29 cemented hips, concluded that a degree of protrusion greater than $5 \mathrm{~mm}$ warranted bone grafting; and that a grossly deficient medial wall required reconstruction with bone graft and additional fixation devices. They also stressed the necessity to restore the normal geometry of the hip joint by placing the cup more laterally. As has been shown, the Ring uncemented prosthesis restores normal loading by its alignment, and that resolution of the pelvic defect occurs as a result. Although perhaps not a simpler surgical technique, the Ring total hip replacement is not time-consuming and it does not require the use of reinforcement implants.

Dysplasia. The value of the dysplastic index is that it allows for comparison of successive radiographs of the same patient. It does not directly correlate with the adequacy of lateral support of the cup; however, a value of one generally indicates a fully supported prosthesis. The CE angle varies somewhat with rotation of the film, but within the ranges used for the grading system, we consider our comparison to be valid. Since the problem is a three-dimensional one, no measurement in two dimensions gives an entirely accurate means of assessment, and the two methods of presenting the results need to be considered together.

Load is transmitted to the lateral edge of the acetabulum, as discussed above, which responds by forming new bone, thus improving lateral support for the cup. This process may continue until the cup is fully covered. In our series the average improvement in the index was $21.5 \%$, and 10 of the 38 hips had an improved grade. 
Conclusion. Although uncemented total hip replacement in the presence of an anatomical defect is technically demanding, the positive response of the pelvic bone contributes to its success. In protrusio acetabuli the medial wall remodels and strengthens, while in the dysplastic acetabulum the lateral support for the Ring UPM acetabular cup must initially be produced by aligning the reamers in a more medial direction. Thereafter, any residual lateral defect tends to resolve.

The resolution in both instances occurs because of the biomechanical design of the prosthesis. The biological process may be further enhanced by bone grafting, but this is rarely necessary.
I am grateful to Mr P. A. Ring for permission to study patients under his care, to Mr P. Shepherd for the photographs and the Medical Illustration Department of The London Hospital for the diagrams.

Bertin KC, Freeman MA, Morscher E, Oeri A, Ring PA. Cementless acetabular replacement using a pegged polyethylene prosthesis. Arch Orthop Trauma Surg 1985:104:251-61.

Ranawat CS, Zahn MG. Role of bone grafting in correction of protrusio acetabuli by total hip arthroplasty. $J$ Arthrop 1986;1:131-7.

Ring PA. Uncemented acetabular replacement. Arch Orthop Trauma Surg 1983;101:225-9.

Sharp DJ, Porter KM, Duke RFN. The resolution of protrusio acetabuli treated with Ring's hip prosthesis. J Bone Joint Surg [Br] 1984:66-B:635-8.

Sotelo-Garza A, Charnley J. The results of Charnley arthroplasty of the hip performed for protrusio acetabuli. Clin Orthop 1978:132:12-8. 